\title{
Comparing galaxy populations in compact and loose groups of galaxies
}

\section{Brightest group galaxies}

\author{
Héctor J. Martínez, Valeria Coenda, and Hernán Muriel
}

\begin{abstract}
Instituto de Astronomía Teórica y Experimental (IATE), CONICET-Observatorio Astronómico, Universidad Nacional de Córdoba, Laprida 854, X5000BGR Córdoba, Argentina

e-mail: julian@oac.uncor.edu
\end{abstract}

Received 21 May 2013 / Accepted 3 August 2013

\begin{abstract}
Aims. The properties of the brightest galaxies (BCGs) are studied in both compact and loose groups of galaxies in order to better understand the physical mechanisms influencing galaxy evolution in different environments.

Methods. Samples of BCGs are selected in compact and loose groups of galaxies previously identified in the Sloan Digital Sky Survey. The following physical properties of the BCGs in compact groups and in sub-samples of loose groups are compared, defined by their mass and total luminosity: absolute magnitude, colour, size, surface brightness, stellar mass, concentration and morphological information from the Galaxy Zoo. The fraction of BCGs classified as red and/or early-type as a function of galaxy luminosity is studied. The fraction of the group's total luminosity contained in the BCG and the difference in luminosity between the BCG and the second-ranked galaxy, are also analysed.

Results. Some properties of BCGs in compact and loose groups are comparable. However, BCGs in compact groups are systematically more concentrated and have larger surface brightness than their counterparts in both, high- and low-mass loose groups. The fractions of red and early-type BCGs in compact groups are consistent with those of high-mass loose groups. Comparing BCGs in sub-samples of compact and loose groups selected for their similar luminosities, BCGs in compact groups are found to be, on average, brighter, more massive, larger, redder and more frequently classified as elliptical. In compact groups, the BCG contains a larger fraction of the system's total luminosity and differs more in absolute magnitude from the second-ranked galaxy. Using a simple model, which dry-merges the BCG in loose groups with a random choice among the 2nd, 3rd and 4th-ranked galaxies in the group, and allowing for some star loss in the process, we show that the absolute magnitude distributions of BCGs in compact and loose groups of similar luminosities can be made more alike.

Conclusions. BCGs in compact and loose groups are found to be different. Some mechanisms responsible for transforming late-type galaxies into early types, such as mergers, may be more effective within compact groups due to their high densities and small velocity dispersion, which would lead their BCGs along somewhat different evolutionary paths.
\end{abstract}

Key words. galaxies: groups: general - galaxies: fundamental parameters - galaxies: evolution

\section{Introduction}

The brightest members of galaxy systems have been the subject of numerous studies. While most of them are concentrated in clusters of galaxies, the brightest galaxies in groups have been less studied, and even fewer studies have focused on the brightest members of compact groups. Brightest cluster galaxies $\left(\mathrm{BCGs}^{1}\right)$ are typically early-type and are among the most massive galaxies known in the Universe. Typically, they have old stellar populations and represent a major challenge for models of galaxy formation and evolution. Cluster BCGs are known to have narrow luminosity and colour distributions (e.g. Postman \& Lauer 1995). Situated in the densest and most extreme environments in the Universe, they may be not just examples of the bright end of the cluster luminosity function, but a different class of objects with their own luminosity function.

It is generally assumed that BCGs acquire most of their stellar mass via dry mergers between smaller halos (De Lucia \& Blaizot 2007), although the low evolution in stellar mass observed for BCGs at different redshifts (Whiley et al. 2008; Collins et al. 2009; Stott et al. 2010) puts strong constraints on

\footnotetext{
1 The acronym will be used generically for both clusters and groups of galaxies.
}

semi-analytic models in the $\Lambda \mathrm{CDM}$ cosmology (see for instance Tonini et al. 2012; Martizzi et al. 2012). The weak evolution of the stellar mass of BCGs with redshift suggests that a large fraction of mergers have happened at high redshift. Mergers at lower redshift are also expected, although their frequency may be a strong function of the environment. Liu et al. (2009) studied the fraction of BCGs that show evidence of ongoing major dry mergers and found that this fraction increases with cluster richness. They suggest that BCG luminosity has increased on average by $15 \%$ since $z \sim 0.7$ due to dry mergers. Edwards \& Patton (2012) analyse a sample of BCGs that have close neighbours and conclude that mergers may have provided up to $10 \%$ of the mass of BCGs since $z \sim 0.3$. Similar results are found by Lidman et al. (2012), but they also suggest that a significant fraction of the mass involved in mergers is lost to the intra-cluster medium (see also Conroy et al. 2007; Stott et al. 2010).

The basic question about BCGs is whether they are special or just the statistical result of selecting the brightest object of a given luminosity function (see Paranjape \& Sheth 2012, and references therein). If mergers are important in the formation of BCGs, this should be reflected in the magnitude gap between the first- and second-ranked galaxies $\left(\Delta M_{12} \equiv M_{2}-M_{1}\right)$. Smith et al. (2010) computed $\Delta M_{12}$ in a sample of clusters and 
found a $3 \sigma$ excess over the prediction from Monte Carlo simulations of a Schechter function that fits the mean luminosity function of cluster galaxies. Although there is a general consensus that BCGs in clusters are special objects, there are some contradictory results for groups of galaxies. Geller \& Postman (1983) studied a sample of groups of galaxies and concluded that their brightest members are less dominant than those in clusters and are consistent with being the luminous tail of the luminosity function (see also Lin et al. 2010; Loh \& Strauss 2006). Nevertheless, Paranjape \& Sheth (2012) found that BCG luminosity distributions in groups are inconsistent with these galaxies having been drawn from a universal luminosity function.

Compact groups are a special type of groups of galaxies: even though they have velocity dispersions comparable to those found in loose groups, they have higher densities, similar to those found in clusters (Hickson et al. 1992), thus providing a different scenario for galaxy mergers. These exceptional conditions may significantly affect the evolution of BCGs in compact groups. The historically small size of the available samples of compact groups has greatly limited statistical study of their brightest members. Díaz-Giménez et al. (2012) used a sample of 78 compact groups and found a large magnitude gap between the first- and second-ranked galaxy. However, they also found that this effect is not present in the samples of compact groups constructed by Hickson et al. (1992), Allam \& Tucker (2000) and Focardi \& Kelm (2002).

Based on the sixth data release of the Sloan Digital Sky Survey (Adelman-McCarthy et al. 2008), McConnachie et al. (2009) identified a large sample of compact groups suitable for detailed statistical studies. With this sample, in Coenda et al. (2012, herafter Paper I) we compared the properties of galaxies in compact and loose groups, using large samples comprising 846 compact groups and 2536 (2528) loose groups of low (high) mass. We found significant differences between the mean properties of galaxies in groups, depending on whether they are in loose or in compact groups. We suggested that the physical mechanisms that transform galaxies into earlier types may be more effective in compact than in loose groups.

This second article of the series is a comparative study of BCG properties in loose and compact groups in the context of the merger scenario. Given the controversial results in the literature for both loose and compact groups, its main purpose is to establish how differences in the dynamic properties of galaxy systems affect the formation and evolution of their brightest galaxies. We also explore whether the differences found in Paper I regarding the overall population of galaxies inhabiting loose and compact groups are also present for BCGs.

The layout of the paper is as follows: in Sect. 2, we describe the samples of groups and galaxies used; we perform a comparative analysis of the BCGs in compact and in loose groups in Sect. 3; finally, our results are summarised and discussed in Sect. 4. Throughout the paper, a flat cosmological model is assumed, with parameters $\Omega_{0}=0.3, \Omega_{\Lambda}=0.7$, and a Hubble's constant $H_{0}=100 \mathrm{~h} \mathrm{~km} \mathrm{~s}^{-1} \mathrm{Mpc}^{-1}$. All magnitudes were corrected for Galactic extinction using the maps by Schlegel et al. (1998) and are in the AB system. Absolute magnitudes and galaxy colours were $K$-corrected using the method of Blanton et al. (2003, KCORRECT version 4.1).

\section{The samples of brightest group galaxies}

As in Paper I, the sample of compact groups used in this paper is drawn from catalogue A of McConnachie et al. (2009), who used the original selection criteria of Hickson (1982) to identify compact groups in the sixth data release of the SDSS (AdelmanMcCarthy et al. 2008). Their catalogue A has 2297 groups, adding up to 9713 galaxies, down to a Petrosian (Petrosian 1976) limiting magnitude of $r=18$, and has spectroscopic information for 4131 galaxies ( $43 \%$ completeness). In order to exclude interlopers, this catalogue includes only groups that have a maximum line-of-sight velocity difference smaller than $1000 \mathrm{~km} \mathrm{~s}^{-1}$. For the purposes of this work, we selected all groups in the A catalogue within the redshift range $0.05 \leq z \leq 0.18$ that have measured spectroscopic redshift for their BCGs. This results in a sample of 477 compact groups.

The samples of loose groups used in this paper were drawn from the sample of groups identified by Zandivarez \& Martínez (2011) in the Main Galaxy Sample (Strauss et al. 2002) of the seventh data release of SDSS (Abazajian et al. 2009) with $14.5 \leq$ $r \leq 17.77$. They used a friends-of-friends algorithm (Huchra \& Geller 1982) to link galaxies into groups, followed by a second identification using a higher density contrast in groups with at least 10 members, in order to split merged systems and clean up any spurious member detection. The authors computed group virial masses from the virial radius of the systems and the velocity dispersion of member galaxies (Limber \& Mathews 1960; Beers et al. 1990). The sample of Zandivarez \& Martínez (2011) comprises 15961 groups with more than 4 members, adding up to 103342 galaxies. It is well-known that the properties of galaxies in groups are correlated with group mass (e.g. Martínez \& Muriel 2006). Thus, as in Paper I, we divided the groups in the Zandivarez \& Martínez (2011) sample into two sub-samples of low, $\log \left(M / M_{\odot} h^{-1}\right) \leq 13.2$, and high, $\log \left(M / M_{\odot} h^{-1}\right) \geq 13.6$, mass. To perform a fair comparison of BCGs, we used a Monte Carlo algorithm to randomly select groups from these two subsamples, in order to construct new sub-samples of low- and highmass loose groups that have redshift distributions similar to that of the compact groups, in the redshift range $0.05 \leq z \leq 0.18$. The final sub-samples of low- and high-mass loose groups include 1414 and 1458 systems, respectively.

Since compact groups in the McConnachie et al. (2009) sample have no measured mass, and in Paper I we were interested in comparing galaxies in samples of compact and loose groups that shared a similar physical magnitude, we used the group total luminosity as a common parameter, and constructed samples of compact and loose groups that have similar luminosities. In this paper, we compare the properties of the BCGs of groups with similar luminosity. Loose group luminosities were computed by Martínez \& Zandivarez (2012) and the luminosities of the compact groups were computed in Paper I, using the method of Moore et al. (1993). By means of the same Monte Carlo algorithm used in Paper I, we constructed two "equal luminosity" sub-samples of compact and loose groups of galaxies that have similar redshift and absolute magnitude distributions within the boundaries $0.05 \leq z \leq 0.15$ and $-20.7 \leq M_{0.1_{r}}^{\text {GROUP }}-5 \log (h) \leq$ -23.8. The equal luminosity sub-samples of compact groups (EQL CGs) and loose groups (EQL LGs) include 314 and 1577 systems, respectively.

\section{Comparing the brightest galaxies of compact and loose groups}

\subsection{Galaxy properties}

This study compares a number of galaxy parameters:

- Petrosian absolute magnitude in the ${ }^{0.1} r$-band;

- the radius that encloses $50 \%$ of the Petrosian flux, $r_{50}$;

- the $r$-band surface brightness, $\mu_{50}$, computed inside $r_{50}$; 


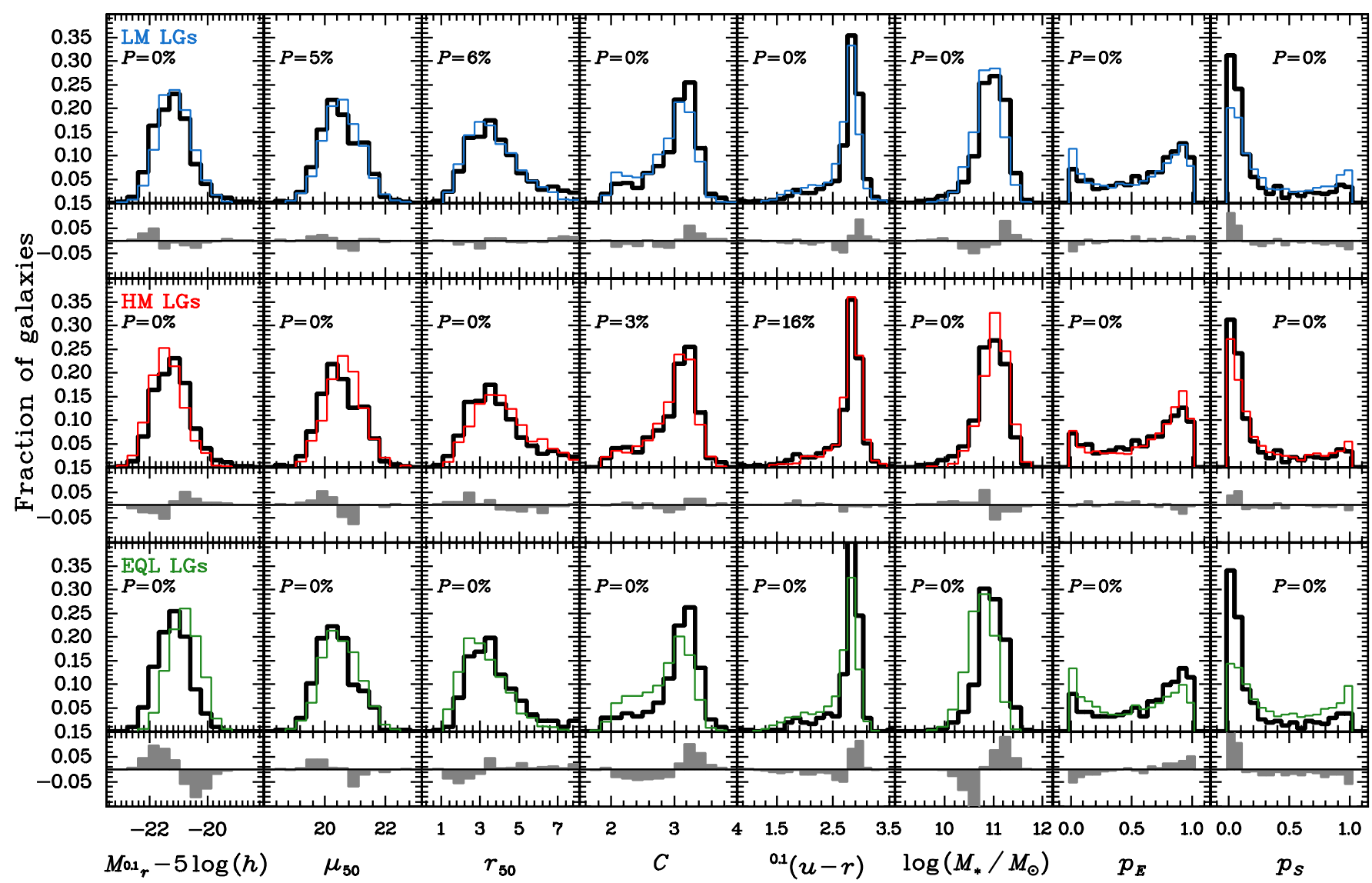

Fig. 1. Distributions of BCG properties, from left to right: ${ }^{0.1} r$-band absolute magnitude; $r$-band surface brightness in mag arcsec ${ }^{-1}$; Petrosian half-light radius in $h^{-1} \mathrm{kpc}$; concentration parameter; ${ }^{0.1}(u-r)$ colour; stellar mass; and the probability of being elliptical $\left(p_{E}\right)$ or spiral $\left(p_{S}\right)$. In the top and middle rows, thick black line corresponds to the sample of compact groups, blue line to low mass loose groups, and red line to high mass loose groups. The bottom row compares the equal luminosity sub-samples of compact (thick black) and loose groups (green). All distributions have been normalised to have the same area. Below each panel, we show as shaded histograms the residuals between the distributions. We quote in each panel the probability of the null hypothesis that both data sets are drawn from the same distribution according to the Kolmogorov-Smirnov test.

- the $r$-band concentration index $C$, defined as the ratio of the radii enclosing 90 and 50 percent of the Petrosian flux;

- the ${ }^{0.1}(u-r)$ colour $^{2}$;

- the stellar mass, $M_{*}$, computed from the absolute magnitude and colour, following Taylor et al. (2011);

- the probability of being elliptical $\left(p_{\mathrm{E}}\right)$ or spiral $\left(p_{\mathrm{S}}\right)$ as measured by the Galaxy Zoo Project ${ }^{3}$ (Lintott et al. 2011).

Figure 1 compares the normalised distributions of BCG parameters in loose and compact groups of galaxies. Below each panel, we show the residuals between each pair of distributions, i.e., for each property $X$, the difference $\Delta F(X)=f_{\mathrm{CG}}(X)-f_{\mathrm{LG}}(X)$, where $f_{\mathrm{CG}}(X)$ and $f_{\mathrm{LG}}(X)$ are the fractions of galaxies in the bin centred on $X$, in the compact and in the loose group sample, respectively. In each panel of this figure, we quote the probability for the null hypothesis that the parameter distributions we compare are drawn from the same distribution according to a Kolmogorov-Smirnov test. The best case is the comparison between the colours of BCGs in compact groups and in high mass loose groups, which reaches only a $16 \%$ probability level.

From the comparison of compact groups and loose groups in the low and high mass sub-samples, we notice that there are

\footnotetext{
2 We use model instead of Petrosian magnitudes to compute colours, since aperture photometry may include non-negligible Poisson and background subtraction uncertainties in the $u$ band.

3 http://zoo1.galaxyzoo.org/
}

two sets of parameters that have different behaviour: for some of them, the properties of the BCGs in compact groups are intermediate between low- and high-mass loose groups, as is the case of absolute magnitude, stellar mass, Petrosian half-light radii, colour and the morphology parameters; on the other hand, according to the two remaining parameters, surface brightness and concentration, the brightest galaxies of compact groups are different from their loose-group counterparts in some more fundamental way. As an example of the first set, if we consider luminosity, BCGs in compact groups are typically brighter than BCGs in low-mass loose groups and fainter than BCGs in highmass loose groups. That is not the case of, for instance, their concentration: galaxies in compact groups are more concentrated than galaxies in loose groups, irrespective of group mass.

Comparison of groups of similar luminosity distributions (bottom row in Fig. 1) reveals differences between BCGs in loose and compact groups in the same sense as the differences between BCGs in low-mass loose groups and compact groups (top row in Fig. 1), albeit more pronounced. Our samples of equal luminosity loose and compact groups have, on average, 4.7 and 3.5 galaxies that are brighter than the $r=17.77$ apparent magnitude limit of the SDSS' Main Galaxy Sample, respectively. That is, at similar group luminosity, loose groups typically have more galaxies than compact groups. In addition, and as we show below (Sect. 3.4), compact groups have a larger luminosity gap between the BCG and the second-ranked galaxy. Thus, 


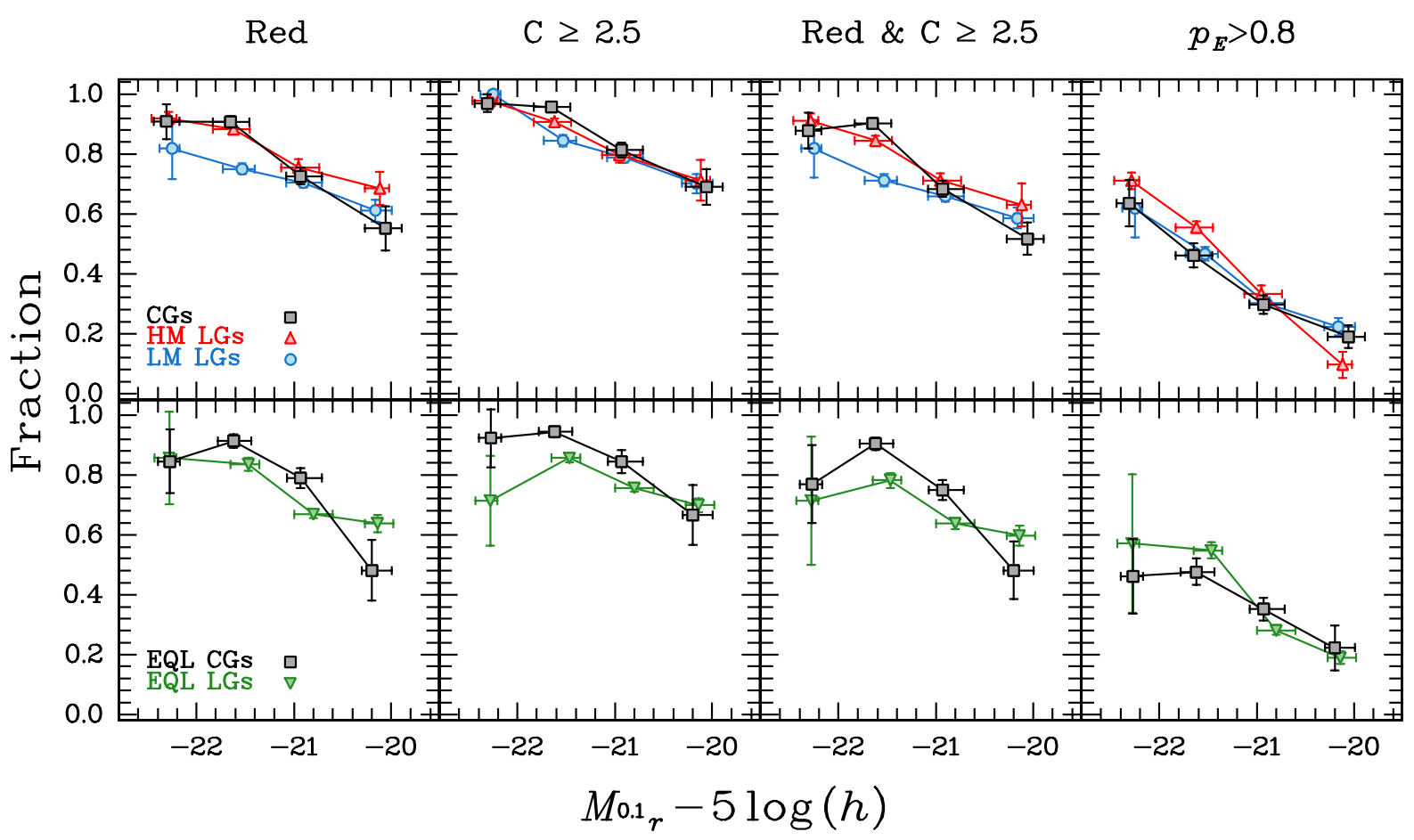

Fig. 2. Left panels: fraction of red BCGs classified according to their ${ }^{0.1}(u-r)$ colour; centre left panels: fraction of early-type BCGs classified according to their concentration parameter; centre right panels: fraction of BCGs classified simultaneously as red and early-type from their colour and concentration parameter; right panels: fraction of BCGs classified as elliptical from their Galaxy Zoo morphology. All fractions are shown as a function of absolute magnitude. Top panels compare compact groups with loose groups of low and high mass, while bottom panels compare compact and loose groups with similar total absolute magnitude. Vertical error-bars are obtained by using the bootstrap resampling technique. Horizontal error-bars are the $25 \%$ and $75 \%$ quartiles of the absolute magnitude distribution within each bin.

selecting a sample of loose groups bound to have a luminosity distribution similar to that of a given sample of compact groups, results in a selection of loose groups with BCGs that are systematically fainter than their compact-group counterparts. Since most galaxy properties are correlated to each other (e.g. Blanton et al. 2005), the fact that the sample of equal-luminosity compact groups have brighter BCGs may provide an explanation for all the differences seen in the bottom row of Fig. 1.

\subsection{The fraction of early-type BCGs}

In clusters of galaxies, BCGs are typically early-type galaxies. In our samples (see Fig. 1), the fraction of BCGs that have a low probability of being elliptical, or the fraction of them that are blue, is not negligible. This sub-section analyses in more detail the BCGs in our group samples by classifying them according to their colour, concentration and Galaxy Zoo morphology.

In Fig. 2 we show the fraction of BCGs as a function of galaxy absolute magnitude that can be classified as early-type according to four different criteria:

i. Colour: we consider that a galaxy belongs to the red sequence if its ${ }^{0.1}(u-r)$ colour is redder than the luminosity dependent threshold of Zandivarez \& Martínez (2011).

ii. Concentration parameter: Strateva et al. (2001) found that the concentration parameter can be used to differentiate between early and late-types. We use the $r$-band concentration index, and consider as early-type galaxies those that have $C \geq 2.5$.

iii. Colour and concentration: we select galaxies that are redder than the luminosity dependent threshold of Zandivarez \& Martínez (2011) and have $C \geq 2.5$. iv. Galaxy Zoo morphology: in addition, we use the morphological classications taken from the Galaxy Zoo Project to classify galaxies as elliptical if $p_{\mathrm{E}}>0.8$. We use the $p_{\mathrm{E}}$ values corrected after the debiasing procedure of Lintott et al. (2011).

The fraction of BCGs that we classify as early-type according to the criteria $\mathrm{i}-\mathrm{iii}$ is similar between compact groups and high mass loose groups and smaller for the low mass sample. Colour is the parameter that distinguishes best between compact groups and low-mass loose groups; classifying galaxies as early-type according to colour and concentration does not give any further information. Thus, according to these results, the fraction of early-type BCGs in compact groups resembles that of the massive loose groups. Comparing groups with similar luminosities shows that compact groups have a larger fraction of early-type galaxies. Within the fraction of BCGs that have a high probability of being elliptical according to the Galaxy Zoo Project (criterion iv), we notice differences: the trend for compact groups is similar to that of low-mass loose groups, and, within errors, the trends for equal-luminosity samples are comparable to each other.

\subsection{The fraction of light in the $B C G$}

Figure 3 shows the distributions of the fraction of group light contained in the brightest galaxy. It is clear from this figure that the brightest group galaxies in compact groups differ markedly from their loose-group counterparts. While they may not be as bright as some BCGs in massive loose groups, in terms of luminosity BCGs in compact groups have a more dominant position within their system. Another conclusion from Fig. 3 is that, in 
Table 1. Mean difference in absolute magnitude between the BCG and the 2nd ranked galaxy and the $T_{1}$ and $T_{2}$ statistics (Tremaine \& Richstone 1977) for our samples of BCGs.

\begin{tabular}{lccccc}
\hline \hline & Compact groups & \multicolumn{2}{c}{ Loose groups } & \multicolumn{2}{c}{ Equal luminosity samples } \\
& & Low mass & High mass & Compact groups & Loose groups \\
\hline \# groups & 477 & 1414 & 1458 & 314 & 1577 \\
$\left\langle\Delta M_{12}\right\rangle$ & $0.68 \pm 0.02^{1}$ & $0.53 \pm 0.01$ & $0.50 \pm 0.01$ & $0.76 \pm 0.03$ & $0.55 \pm 0.01$ \\
$T_{1}$ & $0.95 \pm 0.05$ & $1.08 \pm 0.03$ & $1.17 \pm 0.03$ & $0.74 \pm 0.04$ & $0.96 \pm 0.02$ \\
$T_{2}$ & $0.94 \pm 0.04$ & $1.02 \pm 0.02$ & $1.01 \pm 0.02$ & $0.88 \pm 0.04$ & $1.02 \pm 0.02$ \\
\hline
\end{tabular}

Notes. ${ }^{(1)}$ Quoted errors were computed using the bootstrap resampling technique.

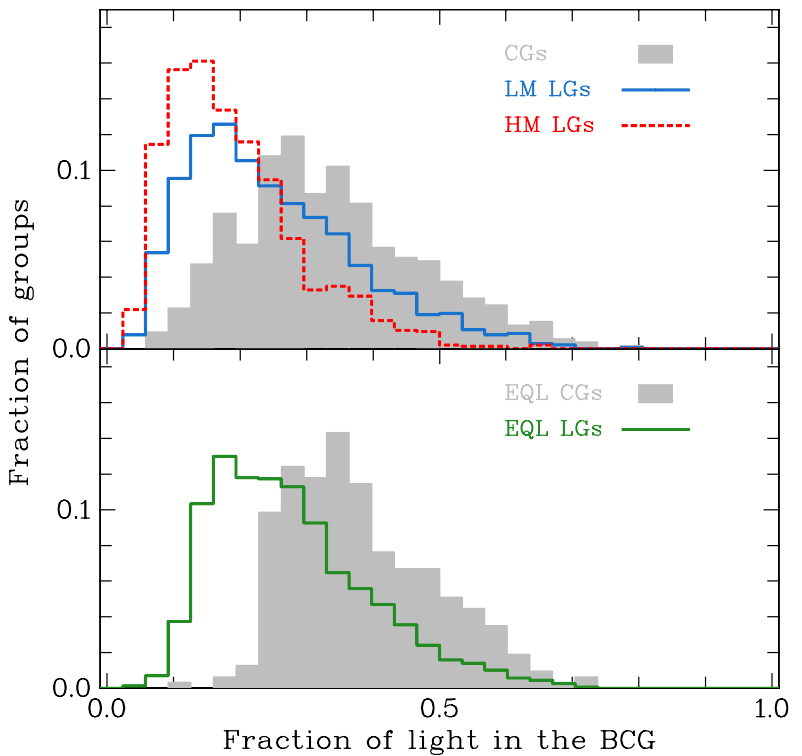

Fig. 3. Distributions of the fraction of group total luminosity contained in the BCG in our samples.

terms of the fraction of light they contain, BCGs in loose groups become less important the more massive the systems that are considered. This agrees with the results by Loh \& Strauss (2006) in clusters of galaxies.

\subsection{Statistical test for BCG luminosities}

The statistical test developed by Tremaine \& Richstone (1977) enables us to distinguish between two possible scenarios for the BCGs: either they are just extreme examples of a luminosity function or, on the contrary, they are objects that may have had a different evolution compared to their companion galaxies. The test is independent of the assumed luminosity and of its variation from cluster to cluster. The key insight of the test is that it makes use of the magnitude difference between the first two ranked galaxies, $\Delta M_{12}$. For a luminosity function with an exponential cut-off at the bright end, $\phi(M) \sim \exp (\alpha M)$, given that BCGs have a small magnitude spread, the difference $\Delta M_{12}$ cannot be excessively large if both galaxies are drawn from the same luminosity function.

Tremaine \& Richstone (1977) defined two parameters:

$T_{1}=\frac{s\left(M_{1}\right)}{\left\langle\Delta M_{12}\right\rangle}$

and

$T_{2}=\frac{s\left(\Delta M_{12}\right)}{0.667^{1 / 2}\left\langle\Delta M_{12}\right\rangle}$ where $\left\langle\Delta M_{12}\right\rangle$ is the mean value of the difference $\Delta M_{12}$, and $s\left(M_{1}\right)$ and $s\left(\Delta M_{12}\right)$ are the standard deviations of $M_{1}$ and $\Delta M_{12}$, respectively. If the first two ranked galaxies are drawn from the same luminosity function, then values of $T_{1} \gtrsim 1$ and $T_{2} \gtrsim 1$ should be expected. Values of $T_{1}$ and $T_{2}$ lower than unity imply that the first-ranked group galaxies are abnormally bright at the expense of the second-ranked galaxy.

We show in Table 1 the mean values of $\left\langle\Delta M_{12}\right\rangle, T_{1}$ and $T_{2}$ for all our samples of groups. The $\left\langle\Delta M_{12}\right\rangle$ value is typically higher for compact groups, irrespective of whether the comparison is made with loose groups of different masses or of similar luminosities. BCGs in compact groups are more luminous relative to their companions. Loose groups in all our samples have values $T_{1}$ and $T_{2}$ similar to or greater than unity, thus consistent with being objects at the bright extreme of the luminosity function of galaxies in groups. This is in agreement with Geller \& Postman (1983) and Lin et al. (2010) but not with the results by Paranjape \& Sheth (2012). On the other hand, $T_{1}$ and $T_{2}$ values are lower for compact groups, below unity but close to it, in between the values obtained by Díaz-Giménez et al. (2012) using their own sample of compact groups and the values computed by the same authors using the samples by Hickson et al. (1992), Allam \& Tucker (2000), Focardi \& Kelm (2002).

\subsection{Dry mergers and BCGs}

The previous sub-sections showed some evidence that BCGs in compact groups are perhaps not just objects in the extreme bright-end of the luminosity function of galaxies, and that their contribution to the parent group total luminosity is larger than the contribution of loose groups' BCGs to their own systems. In this sub-section, we explore whether the BCGs in compact groups have grown brighter at the expense of some of their companions, by means of a toy model in which we take loose groups and brighten their BCGs by dry-merging them with another galaxy in the group. We will use the term dry merger to name mergers in which there is no star formation, regardless of whether the galaxies involved are early or late types. In our model, even when one or both of the galaxies undergoing a merging process may still have gas available, we will assume for simplicity's sake that there is no star formation. For this purpose, we use samples of groups selected as having similar luminosity distributions, in order to perform comparisons between systems sharing a similar physical magnitude.

In this very simple scheme, for every group in the EQL LG sample, we model the dry merging of its BCG with the $i$-th ranked galaxy by summing their $r$-band luminosities, in order to brighten the BCG at the expense of that companion. We also consider the possibility of stellar mass losses in the merger process by subtracting a randomly chosen fraction of the fainter galaxy's luminosity. This fraction is allowed to be as high as 30\%. Even if 


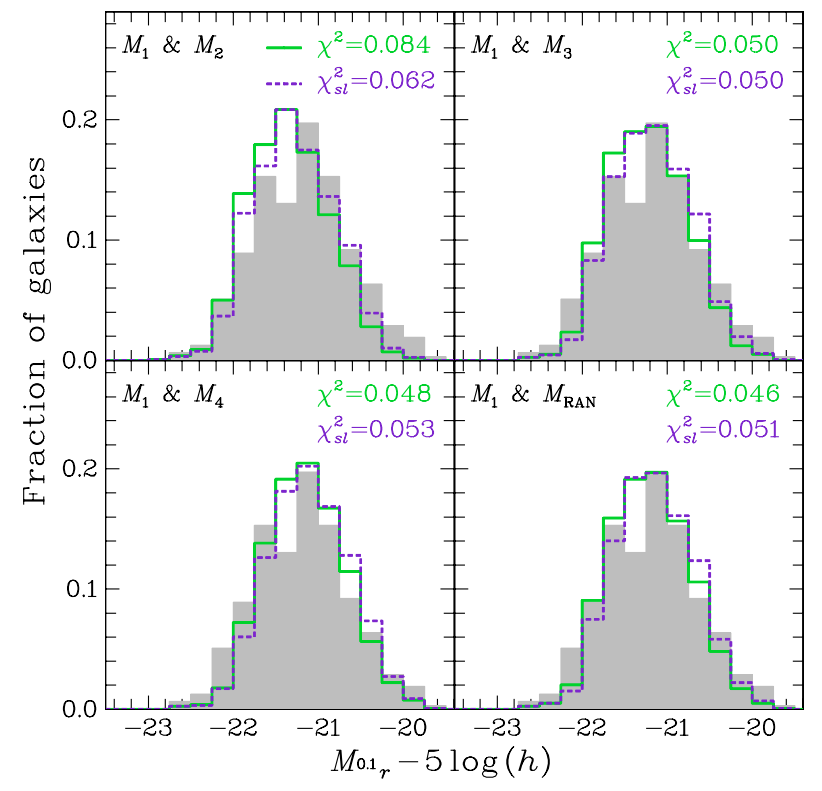

Fig. 4. Distributions of BCG absolute magnitudes. Shaded areas: BCGs in the EQL CG sample. We show in the continuous green line the distribution of absolute magnitudes resulting from the combined luminosity of the EQL LG BCG and: the second brightest galaxy in the group (top left panel); the third brightest galaxy (top right panel); the fourth brightest galaxy (bottom left panel); and a galaxy randomly chosen among the 2nd-, 3rd- and 4th-ranked galaxies. The distributions shown in the dashed violet line, are similar to the green ones, but we allow for an up to $30 \%$ randomly chosen fraction of stars to be lost in the merging process. We quote in each panel the $\chi^{2}$ statistics of the comparison between the models and the shaded distribution.

the stars lost during the merger process remain at the bottom of the potential well or form a halo around the BCG, the standard photometry of the SDSS cannot account for this excess of light (see Tal \& van Dokkum 2011). Therefore, in our toy model we assume that the star loss during a merger also represents a loss in total group luminosity.

Figure 4 shows the absolute magnitude distribution of the galaxies resulting from the dry merging of the BCG $\left(M_{1}\right)$ with, alternatively, the 2 nd $\left(M_{2}\right), 3 \mathrm{rd}\left(M_{3}\right)$ and 4 th $\left(M_{4}\right)$ ranked galaxy. We also show the result of merging the BCG with a galaxy randomly chosen $\left(M_{\text {RAN }}\right)$ among $M_{2}, M_{3}$ and $M_{4}$. In all cases, we show the results with and without star loss and quote the $\chi^{2}$ values that result from comparing the model with the sample of EQL CGs. When no star loss is considered, the best match between the absolute magnitude distribution of the model galaxies and the EQL CG BCGs is obtained when merging the BCG with the fourth-ranked galaxy. When we allow up to $30 \%$ star loss in the merging, the best match occurs when the BCG merges with the third-ranked galaxy.

A detailed characterisation of the processes involved in the formation of the brightest galaxies in compact groups is far beyond the scope of our model. However, we can infer from the model that BCGs in compact groups may have had more mergers in their past history than their counterparts in loose groups, regardless of which of the former group members were involved in the merging process.

\section{Discussion and conclusions}

We study the properties of the brightest galaxies in compact and loose groups of galaxies to deepen our understanding of the physical mechanisms acting upon galaxy evolution in different environments.

We select samples of BCGs in compact groups drawn from McConnachie et al. (2009), and in loose groups taken from Zandivarez \& Martínez (2011). A number of physical properties of the BCGs are compared in compact groups and in subsamples of loose groups defined by their mass and total luminosity, namely: absolute magnitude, colour, size, surface brightness, stellar mass, concentration as well as morphological information from the Galaxy Zoo. We also study the fraction of BCGs that are classified as red and/or early-type as a function of galaxy luminosity. We analyse the fraction of the group's total luminosity contained in the BCG and the difference in luminosity between the brightest and the second-ranked galaxies.

Some properties of the BCGs in compact groups are comparable to those of BCGs in average loose groups. However, BCGs in compact groups are systematically more concentrated and have a larger surface brightness than their counterparts in both high- and low-mass loose groups. The fractions of red and early-type BCGs in compact and high mass loose groups are consistent with each other. Comparing BCGs in sub-samples of compact and loose groups selected to have similar luminosities, we find that BCGs in compact groups are, on average, brighter, more massive, larger, redder and more frequently classified as elliptical.

Compared to BCGs in loose groups, BCGs in compact groups are found to contain a larger fraction of the system's total luminosity and differ more in absolute magnitude from the second-ranked galaxy. Using a simple model, in which we drymerge the BCG in loose groups with another, randomly chosen, galaxy in the group, and allowing for some star lossin the process, we show that the absolute magnitude distributions of BCGs in compact and loose groups of similar luminosities can be made more alike.

We have shown in a previous work (Paper I), that the overall galaxy population in compact groups has undergone a major transformation compared to loose-group galaxies. In this work we find that their BCGs also differ. Some mechanisms responsible for transforming late-type galaxies into early types, such as mergers, may be more effective within compact groups due to their high densities and small velocity dispersion, thus leading their BCGs along somewhat different evolutionary paths.

From our analyses of the fraction of group light in the BCG and the difference in absolute magnitude relative to the second ranked galaxy, it is clear that BCGs in compact groups are more luminous compared to their companions or to their parent group than BCGs in loose groups.

We find values of the Tremaine \& Richstone statistics for compact groups that are slightly below unity, which may indicate that their BCGs are not completely consistent with being part of the bright end of the luminosity function for galaxies in groups. Our measurements of $T_{1}$ and $T_{2}$ are in between values found in previous works, below unity but close to it, higher than the values obtained by Díaz-Giménez et al. (2012), and smaller than, although closer to, the results the same authors obtained from the samples by Hickson et al. (1992), Allam \& Tucker (2000), and Focardi \& Kelm (2002).

On the other hand, BCGs in our loose-group samples are clearly drawn from the bright end of the luminosity function, in agreement with Geller \& Postman (1983). These facts may be an indication of the different evolution of central galaxies in compact groups.

In a hierarchical scenario for galaxy formation and evolution, mergers play a key role in the building up of galaxies. 


\section{H. J. Martínez et al.: Brightest group galaxies}

Galaxies get bigger by accretion and by merging with other galaxies. Given their isolation, compact groups are not likely to have had much available material in their surroundings to accrete. In particular, to explain their prominent luminosity relative to the system they inhabit, BCGs in compact groups may have had a merger history that contributed very efficiently to their growth in luminosity/mass compared to their companion galaxies. Mergers that built up the central galaxies in compact groups may have been more efficient per unit of group luminosity/mass than mergers that originated the central galaxies in loose groups.

Acknowledgements. This work was supported with grants from CONICET (PIP 11220080102603 and 11220100100336); Ministerio de Ciencia y Tecnología de la Provincia de Córdoba, Argentina (PID 2008/14797627); and Secretaría de Ciencia y Tecnología Universidad Nacional de Córdoba, Argentina. Funding for the Sloan Digital Sky Survey (SDSS) was provided by the Alfred P. Sloan Foundation, the Participating Institutions, the National Aeronautics and Space Administration, the National Science Foundation, the U.S. Department of Energy, the Japanese Monbukagakusho, and the Max Planck Society. The SDSS Web site is http://www.sdss.org/. The SDSS is managed by the Astrophysical Research Consortium (ARC) for the Participating Institutions. The participating institutions are the University of Chicago, Fermilab, the Institute for Advanced Study, the Japan Participation Group, the Johns Hopkins University, the Korean Scientist Group, Los Alamos National Laboratory, the Max Planck Institut für Astronomie (MPIA), the Max Planck Institut für Astrophysik (MPA), New Mexico State University, University of Pittsburgh, University of Portsmouth, Princeton University, the United States Naval Observatory, and the University of Washington.

\section{References}

Abazajian, K. N., Adelman-McCarthy, J. K., Agüeros, M. A., et al. 2009, ApJS, 182,543

Adelman-McCarthy, J. K., Agüeros, M. A., Allam, S. S., et al. 2008, ApJS, 175, 297

Allam, S. S., \& Tucker, D. L. 2000, Astron. Nachr., 321, 101

Beers, T. C., Flynn, K., \& Gebhardt, K. 1990, AJ, 100, 32

Blanton, M. R., Brinkmann, J., Csabai, I., et al. 2003, AJ, 125, 2348
Blanton, M. R., Eisenstein, D., Hogg, D. W., Schlegel, D. J., \& Brinkmann, J. 2005, ApJ, 629, 143

Coenda, V., Muriel, H., \& Martínez, H. J. 2012, A\&A, 543, A119

Collins, C. A., Stott, J. P., Hilton, M., et al. 2009, Nature, 458, 603

Conroy, C., Wechsler, R. H., \& Kravtsov, A. V. 2007, ApJ, 668, 826

De Lucia, G., \& Blaizot, J. 2007, MNRAS, 375, 2

Díaz-Giménez, E., Mamon, G. A., Pacheco, M., Mendes de Oliveira, C., \& Alonso, M. V. 2012, MNRAS, 426, 296

Edwards, L. O. V., \& Patton, D. R. 2012, MNRAS, 425, 287

Focardi, P., \& Kelm, B. 2002, A\&A, 391, 35

Geller, M. J., \& Postman, M. 1983, ApJ, 274, 31

Hickson, P. 1982, ApJ, 255, 382

Hickson, P., Mendes de Oliveira, C., Huchra, J. P., \& Palumbo, G. G. 1992, ApJ, 399,353

Huchra, J. P., \& Geller, M. J. 1982, ApJ, 257, 423

Lidman, C., Suherli, J., Muzzin, A., et al. 2012, MNRAS, 427, 550

Limber, D. N., \& Mathews, W. G. 1960, ApJ, 132, 286

Lin, Y.-T., Ostriker, J. P., \& Miller, C. J. 2010, ApJ, 715, 1486

Lintott, C., Schawinski, K., Bamford, S., et al. 2011, MNRAS, 410, 166

Liu, F. S., Mao, S., Deng, Z. G., Xia, X. Y., \& Wen, Z. L. 2009, MNRAS, 396 2003

Loh, Y.-S., \& Strauss, M. A. 2006, MNRAS, 366, 373

Martínez, H. J., \& Muriel, H. 2006, MNRAS, 370, 1003

Martínez, H. J., \& Zandivarez, A. 2012, MNRAS, 419, L24

Martizzi, D., Teyssier, R., \& Moore, B. 2012, MNRAS, 420, 2859

McConnachie, A. W., Patton, D. R., Ellison, S. L., \& Simard, L. 2009, MNRAS, 395,255

Moore, B., Frenk, C. S., \& White, S. D. M. 1993, MNRAS, 261, 827

Paranjape, A., \& Sheth, R. K. 2012, MNRAS, 423, 1845

Petrosian, V. 1976, ApJ, 209, L1

Postman, M., \& Lauer, T. R. 1995, ApJ, 440, 28

Schlegel, D. J., Finkbeiner, D. P., \& Davis, M. 1998, ApJ, 500, 525

Smith, G. P., Khosroshahi, H. G., Dariush, A., et al. 2010, MNRAS, 409, 169

Stott, J. P., Collins, C. A., Sahlén, M., et al. 2010, ApJ, 718, 23

Strateva, I., Ivezić, Ž., Knapp, G. R., et al. 2001, AJ, 122, 1861

Strauss, M. A., Weinberg, D. H., Lupton, R. H., et al. 2002, AJ, 124, 1810

Tal, T., \& van Dokkum, P. G. 2011, ApJ, 731, 89

Taylor, E. N., Hopkins, A. M., Baldry, I. K., et al. 2011, MNRAS, 418, 1587

Tonini, C., Bernyk, M., Croton, D., Maraston, C., \& Thomas, D. 2012, ApJ, 759 43

Tremaine, S. D., \& Richstone, D. O. 1977, ApJ, 212, 311

Whiley, I. M., Aragón-Salamanca, A., De Lucia, G., et al. 2008, MNRAS, 387, 1253

Zandivarez, A., \& Martínez, H. J. 2011, MNRAS, 415, 2553 